\title{
Reflection and Transmission Coefficients from Rectangular Notches in Pipes
}

\author{
Darryl K. Stoyko ${ }^{1,2, *}$ - Neil Popplewell ${ }^{2}$ - Arvind H. Shah ${ }^{3}$ \\ 1 Stress Engineering Services Canada \\ 2 University of Manitoba, Department of Mechanical and Manufacturing Engineering, Canada \\ 3 University of Manitoba, Department of Civil Engineering, Canada
}

The use of a single, non-dispersive ultrasonic guided wave mode is one important approach to monitoring a structure's health. It is advantageously non-destructive with the ability of propagating over tens of metres to detect a hidden defect. The dimensional assessment of a defect, on the other hand, requires reflection coefficients for two or more such modes. Multiple modes may be excited simultaneously by applying a short pulse to a structure's external surface. This situation is examined here for a circular, hollow and homogeneous, isotropic pipe having negligible damping and an open rectangular notch. A finite element model is employed in a region around a notch. It is coupled to a wave function expansion in the two adjacent, effectively, semi-infinite pipes. Representative longitudinal and flexural modes are investigated for different notch dimensions. A nonaxisymmetric notch, unlike an axisymmetric notch, introduces a plethora of cross modal couplings that lead to more singularities in a reflection coefficient's frequency dependence. There is, however, a common pattern to these distinctive singularities. It is conjectured that singularities corresponding to propagating modes may enable a notch to be detected and its dimensions determined.

Keywords: pipe, notch, cutoff frequency, singularity, guided waves

\section{INTRODUCTION}

Pipelines are used ubiquitously to transport fluids. For example, the Alberta Energy and Utilities Board (EUB) reports that a total of $377,248 \mathrm{~km}$ of energy related pipeline was under its jurisdiction at the end of 2005. The same source also indicates there were a total of 12,848 pipeline "incidents" between 1990 and 2005 . About $95 \%$ of the reported incidents led to a pipeline leak or rupture. Hence, it is clear that extensive networks of pipelines are in widespread use and they are prone to occasional failure. Clearly a method of inspecting pipelines is required to detect and size defects and, ideally, it should be non-invasive.

Guided waves are appealing because they can propagate over long distances, say tens of metres, and they are capable of rapidly interrogating entire structures, including otherwise inaccessible regions. A thorough literature review of guided wave inspection of pipes may be found in [1], so only references pertinent to the present work are given here. Early attempts of using guided waves for pipe inspection focussed on the torsional and longitudinal wave modes and considered spurious reflections as an indication of damage. More recent work has focussed also on reflections of axisymmetric pipe modes from defects. The use of flexural waves has been infrequent because "the acoustic field is much more complicated than the case of axisymmetric modes" [2]. On the other hand, the identification of spatially decaying modes, which are introduced in a pipe by a notch and are analogous to end modes [3], has not been reported.

The first objective of this paper is to demonstrate that singularities, where the term singularity is used to indicate a frequency at which a displacement response of a given guided wave mode becomes very large and behaves similarly to a resonant frequency of an undamped single degree of freedom oscillator, distinct from an unblemished pipe's cutoff frequencies are present when a notch is introduced. These singularities are analogous, in some sense, to the end modes reported in [3]. A second objective is to describe a technique that takes advantage of these singularities to characterise the dimensions of an axisymmetric notch in a pipe. The last objective is to suggest that the extension to nonaxisymmetric and more general notch geometries is straightforward but computationally expensive.

The proposed technique to detect and characterise the dimensions of an axisymmetric notch has a number of advantages. It utilizes the classical and simplest means, i.e., a radial point load acting on the pipe's outer surface, of introducing ultrasonic energy into a pipe to simultaneously excite a number of modes. The notch may be detected by simply examining the spectral density or reflection coefficient of the response. As frequency differences are used to infer a notch's dimensions, the need for consistent transducer coupling is reduced somewhat compared to methods that make use of amplitude changes. A single measurement can yield sufficient information to 
determine a notch's dimensions. This attribute offers an advantage over methods that rely on the excitation of a single mode to determine a reflection coefficient, say, as at least two modes are required to uniquely determine even an axisymmetric notch's dimensions [4]. However, because the proposed method makes use of measurements relatively close to a notch, where waves incident and scattered by the notch may interact (in the reflected field), only modest lengths of pipe are required. Consequently, the procedure can be applied only locally to a notch as waves are used whose amplitudes decay exponentially from a notch's boundary. The technique complements, therefore, other methods that can rapidly screen long lengths of pipe.

The newly discerned singularities are demonstrated to exist first by applying the hybrid semianalytical finite element (SAFE) in combination with a standard finite element procedure to axisymmetric notches in a (hollow) steel pipe. The pipe is assumed to be homogeneous, linearly elastic, isotropic, and uniformly right circular. A parametric study is undertaken subsequently in which the radial depth and axial extent of outer surface breaking, rectangular axisymmetric notches are varied independently. The results are used to illustrate how the frequency differences between the new singularities and an unblemished pipe's cutoff frequencies can be used to detect a notch and determine its size. Solely outer surface breaking notches are considered because the simulations can be partially corroborated by existing experimental data [4]. The examples suggest that almost any set, which contains a sufficient number of accurate frequency differences, will give the same inverse solution. The modes could be selected generally but they are selected usually on the basis of ease of experimental implementation. The extension to nonaxisymmetric notches is suggested by showing that the singularities still exist and characteristic behaviours can be generalized.

\section{HYBRID SAFE AND STANDARD FINITE ELEMENT PROCEDURE}

\subsection{Overview of Hybrid Wave Function-Standard Finite Element Approach}

A hybrid wave function-standard finite element approach employs a conventional finite element description to model the displacement field in a region completely enclosing a nonhomogeneity. The displacement field in the remainder of the waveguide is described in terms of a modal expansion of the "parent" waveguide's wave functions. (References pertinent to pipes are given in [1].) An incident wave field is generated in the parent waveguide. Waves are scattered by the nonhomogeneity and the corresponding (scattered) wave field is obtained by enforcing continuity (displacements and stresses/ nodal forces) between the finite element and wave function expansion regions.

Fig. 1a shows standard orthographic views of a pipe having a nonaxisymmetric, outer surface breaking notch. The nonhomogeneity in this case is the notch. It is bounded by the planes $z=0$ and $z=-2 z_{\mathrm{FE}}$, which demarcate the axial extents of the finite element region. An approximate wave function expansion is used outside this region. The incident wave field is generated by the transient input excitation, shown as a radial point force in the figure, applied in the $z=z_{\mathrm{L}}$ plane. The combined incident and reflected (transmitted) wave field in the parent wave guide corresponds to $z \geq 0\left(z \leq-2 z_{\mathrm{FE}}\right)$, in the configuration shown. Note that the notched pipe shown is symmetric about the plane $z=-z_{\mathrm{FE}}$ which corresponds to the finite element boundary $B^{-}$. Computationally advantageous use is made of this symmetry by decomposing the input excitation into the sum of a pair of forces that are symmetric and antisymmetric about the plane $z=-z_{\mathrm{FE}}$. Then boundary conditions appropriate for symmetric and antisymmetric loadings can be applied to the finite element boundary $B^{-}$. Moreover, the displacement field in the waveguide need be computed only for $z \geq 0$, i.e., the reflected field, because the wave field in the transmitted field can be obtained from the reflected field by applying symmetric and antisymmetric arguments. Note that symmetry is not required to employ the hybrid wave function-standard finite element technique. Geometries that do not possess a plane of symmetry can be accommodated by enforcing continuity conditions between the finite element region and wave guide on two cross sections. The computational effort is increased, however.

Three components are required to apply the hybrid wave function-standard finite element technique. They are: i) the wave functions of the parent waveguide, ii) a finite element description of the region enclosing the defect, and iii) a method of enforcing continuity conditions between the first two components. Each component is described briefly now.

\subsection{SAFE Modelling for Pipes}

The SAFE formulation, detailed exhaustively in [1], provides an easily applied and accurate numerical 
a)

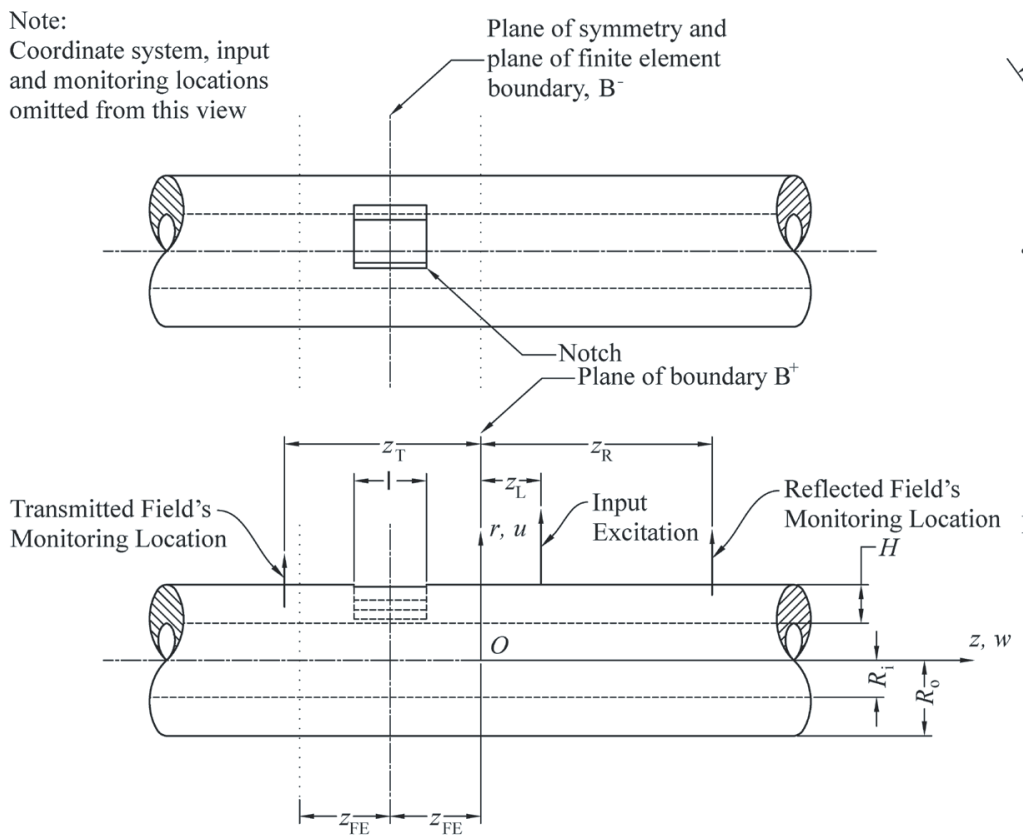

b)

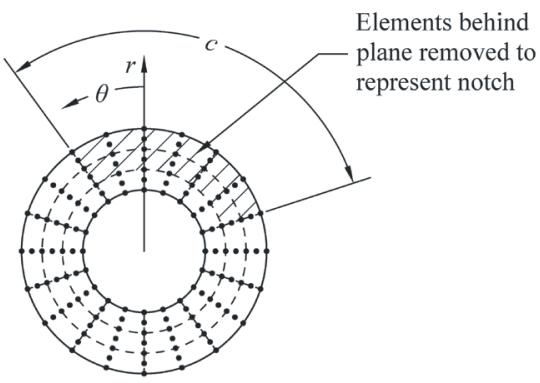

Fig. 1. Illustrating; a) a nonaxisymmetrically notched pipe and b) the finite element nodal points on boundary $B^{+}$which is located at $z=0$; point " $O$ " is the origin of the cylindrical coordinate system

model by which the Green's and wave functions of a pipe may be computed for a harmonic excitation having circular frequency $\omega$. The frequency $\omega$ may be chosen arbitrarily so that an arbitrarily fine frequency resolution may be achieved computationally. This methodology is adapted here to compute the wave functions of a homogeneous, isotropic pipe subjected to a transient excitation. The transient excitation is decomposed into an infinite number of discrete frequency components by using a Fourier transform. Each frequency component of a point force is approximated by using a "narrow" pulse having a uniform amplitude of $\left(2 r_{0} \theta_{0}\right)^{-1}$ over a circumferential distance $2 r_{0} \theta_{0}$ to circumvent convergence difficulties. The $r_{0}$ is the radial coordinate where the point-like force is applied, while $\theta_{0}$ is the angle over which the pulse acts. This narrow pulse is represented, in turn, by employing a Fourier series, in the circumferential direction, of "ring-like" loads having separable spatial and time, $t$, variations.

The pipe is discretized by using $N$ layers through its thickness. (The layers are taken to have identical thicknesses here.) Each layer corresponds to a one-dimensional finite element in the pipe's radial direction for which a quadratic displacement interpolation function is assumed. A finite element approach is applied, layer by layer, in SAFE to approximate the elastic equations of motion. The displacement is represented, like the excitation, by a Fourier series in the circumferential coordinate.

The Fourier series describing the excitation and displacement are substituted into approximate equations of motion obtained from Hamilton's principle. The result is transformed into the wavenumber domain by applying the Fourier integral transform. Then the $n^{\text {th }}$ circumferential harmonic (wave number) takes the form of a quadratic eigensystem which is linearized for the special case when no excitation is applied. Note that the circumferential wave numbers of a right circular pipe can take only integer values due to the requirement that the displacement field should be single valued. The resulting eigenvalues, $k_{n m}$, and (right) eigenvectors, $\phi_{n m}^{\mathrm{R}}$, of this eigensystem are the approximate axial wave numbers and modes shapes through the thickness, respectively, for the $n^{\text {th }}$ circumferential harmonic. The index $m$ is an integer value that is used to indicate the $m^{\text {th }}$ axial mode corresponding to the $n^{\text {th }}$ circumferential wave number. Modes are labelled using the standard convention described in [6]. Both the displacement (response) and excitation, for the $n^{\text {th }}$ circumferential harmonic, are expanded into a series of the normal modes (wave functions) of the linearized eigensystem. A displacement is obtained, for the $n^{\text {th }}$ 
circumferential wave-number and those axial cross sections having positive or negative $z$, by linearly superimposing the appropriate admissible $6 N+3$ right eigenvector solutions. Applying first the inverse Fourier transform to this sum, and then Cauchy's residue theorem, produces the $n^{\text {th }}$ circumferential mode of the displacement. A linear superposition of the circumferential harmonics gives the displacement for a harmonic component of the excitation. The total displacement produced by a multi-frequency excitation is found by linearly superimposing the displacements caused by each individual frequency component. See [1] for further details.

\subsection{Finite Element Idealization}

The finite element method is a well understood tool that is in common use. Therefore, only an outline pertinent to implementing the hybrid wave functionstandard finite element technique is provided. Further pertinent details may be found in [1].

Hamilton's principle is first applied straightforwardly in the finite element region immediately surrounding the notch. To represent a notch, elements are simply removed from the finite element mesh. See Fig. 1b. It is well understood that singularities in the stress field that occur at the notch's corners are not described accurately by this method. However, the far field behaviour is modelled with sufficient accuracy to be meaningful.

The equations of motion of the finite element region are partitioned first such that components related to the interior nodes, where no external forces are applied, can be condensed out so that only quantities on the boundaries $B^{+}$and $B^{-}$remain. Then advantage is taken of the symmetry of the notched pipe about the plane $z=-z_{\mathrm{FE}}$ in Fig. 1a. For (anti) symmetric loading, the displacement in the $(r$ and $\theta$ ) $z$ direction(s), as well stress $\left(\sigma_{z z}\right) \sigma_{r z}$ and $\sigma_{\theta z}$ and corresponding nodal forces vanish on $B^{-}$. The zero displacements on $B^{-}$are condensed out and the unknown reaction forces are ignored (as they are not presently of interest).

Equations are produced that contain a known dynamic stiffness matrix and, as yet, unknown finite element nodal displacements and forces on boundary $B^{+}$. These unknown displacements and forces are written in terms of the unblemished pipe's wave functions for two cases. The first case is when the notch is axisymmetric, i.e., $c$ in Fig. 1a is $360^{\circ}(2 \pi)$; the second, nonaxisymmetric case is when $c$ is less than $360^{\circ}(2 \pi)$. Note that a notch may have a depth, $d$, which is zero and represents an unblemished pipe.
This important case is considered in the transparency check discussed later.

\subsection{Interface between the Wave Function and Finite Element Regions}

A single incident wave mode of unit magnitude that is incident on the plane $z=0$ is considered for simplicity. The scattering caused by an arbitrary incident wave field may be constructed by appropriately scaling and superimposing the scattered wave fields calculated for all the modes present in the incident field. For the sake of discussion, let the incident wave be time harmonic with circular frequency $\omega$ and have a circumferential wave number $n_{\text {in }}$, with an axial wave number $k_{n_{\mathrm{in}} m_{\mathrm{in}}}$. Note that only modes having a non-positive imaginary component to their axial wave number are admissible in the incident field for the configuration shown in Fig. 1a. This restriction is due to the radiation condition that requires the displacement field to remain bounded at $z= \pm \infty$.

It is appropriate to use axisymmetric elements in the finite element region when the notch is axisymmetric. Then the finite element nodal points on the boundary $B^{+}$, as shown in Fig. $1 \mathrm{~b}$, lie on the single radial line $\theta=0$. Note that, because the parent waveguide and the finite element region are both axisymmetric, the circumferential wave number of the scattered waves is required to be identical to that of the incident waves. See, for example, [7].

The finite element region is chosen in the axisymmetric case such that its axial boundaries correspond to those of the notch, i.e., the finite element region is bounded by the planes $z=-l$ and $z=0$. The plane of symmetry is then $z=-l / 2$, and $-2 z_{\mathrm{FE}}=l$. This choice simultaneously simplifies the computations and reduces the number of finite elements used in the idealization.

Using modal superposition, the displacements at $z=0$ of the reflected wave field caused by the incident wave can be written at the finite element nodal points along the radial line $\theta=0$ in the form:

$$
\mathbf{q}_{+}^{\mathrm{s}}=\sum_{m_{\mathrm{s}}=1}^{6 N+3} A_{n_{\mathrm{s}} m_{\mathrm{s}}} \boldsymbol{\phi}_{n_{s}}^{\mathrm{R}} m_{\mathrm{s}},
$$

where the sub, subscript s indicates the scattered wave field, $n_{\mathrm{s}}=n_{\mathrm{in}}, A_{n_{\mathrm{s}} m_{\mathrm{s}}}$ and $\phi_{n_{\mathrm{s}} m_{\mathrm{s}}}^{\mathrm{R}}$ are the amplitude and mode shape of the $n_{\mathrm{s}} m_{\mathrm{s}}^{\text {th }}$ scattered mode. (Note that only modes having non-negative, imaginary wave number components are admissible in the reflected wave field.) Continuity constraints between the wave 
function and finite element regions are applied on the nodal forces and displacements on boundary $B^{+}$.

The (consistent) force vector at the points along the radial line $\theta=0$ can be obtained, for the incident and reflected wave fields by expressing the modal stresses in terms of the displacement wave functions and integrating the product of the stresses and the finite element shape functions over the surface of each finite element. Amplitudes of the scattered waves are found straightforwardly from an invertible matrix equation that results from algebraically rearranging the equations of motion that result from applying the continuity constraints. The matrix equation is invertible because all the approximate modes are retained in the modal expansion and the number of modes is identical to the number of constraint equations. This equation must be evaluated for both the symmetric and antisymmetric components of the load, by changing the boundary conditions on $B^{-}$, in order to recover their combined effects. At any location, the reflected $(z \geq 0)$ and transmitted $\left(z \leq-2 z_{\mathrm{FE}}\right)$ wave amplitudes for the $n_{\mathrm{s}} m_{\mathrm{s}}^{\text {th }}$ scattered mode, $R_{n_{\mathrm{s}} m_{\mathrm{s}}}$ and $T_{n_{\mathrm{s}} m_{\mathrm{s}}}$ respectively, due to a given incident mode, are given by

$$
R_{n_{\mathrm{s}} m_{\mathrm{s}}}=\left(A_{n_{\mathrm{s}} m_{\mathrm{s}}}^{\mathrm{s}}+A_{n_{\mathrm{s}} m_{\mathrm{s}}}^{\mathrm{a}}\right) / 2,
$$

and

$$
T_{n_{\mathrm{s}} m_{\mathrm{s}}}=\left(A_{n_{\mathrm{s}} m_{\mathrm{s}}}^{\mathrm{s}}-A_{n_{\mathrm{s}} m_{\mathrm{s}}}^{\mathrm{a}}\right) / 2 .
$$

The $A_{n_{\mathrm{s}} m_{\mathrm{s}}}$ is the $n_{\mathrm{s}} m_{\mathrm{s}}^{\text {th }}$ scattered wave amplitude and superscript (a) $\mathrm{s}$ denotes the solution corresponding to the (anti) symmetric boundary conditions. $\quad R_{n_{\mathrm{s}} m_{\mathrm{s}}}$ and $T_{n_{\mathrm{s}} m_{\mathrm{s}}}$ represent normalized reflection and transmission coefficients, respectively, because they are calculated by assuming a single incident mode having a unit amplitude. Note that the magnitudes of $R_{n_{\mathrm{s}} m_{\mathrm{s}}}$ and $T_{n_{\mathrm{s}} m_{\mathrm{s}}}$ depend on the scaling of the mode shapes; all mode shapes are scaled here to have a vector norm magnitude of unity. Moreover, the $R_{n_{s} m_{\mathrm{s}}}$ and $T_{n_{s} m_{\mathrm{s}}}$ represent the amplitudes of the scattered waves at the planes $z=0$ and $z \leq-2 z_{\mathrm{FE}}$, respectively.

The procedure for the nonaxisymmetric notch is essentially identical to before but with two major differences. First, all the circumferential wave numbers used in the modal expansion participate, in principle, in the reflected displacement field even though a single incident wave is assumed. Therefore, threedimensional elements are required now in the finite element region and clearly finite element nodal points on the boundary $B^{+}$have to be arranged around this entire boundary, as shown in Fig. 1b. Consequently
Eq. (1) becomes:

$$
\mathbf{q}_{+}^{\mathrm{s}}=\sum_{n_{\mathrm{s}}=n_{\min }}^{n_{\max }} \sum_{m_{\mathrm{s}}=1}^{6 N+3} A_{n_{s} m_{\mathrm{s}}} \boldsymbol{\phi}_{n_{\mathrm{s}} m_{\mathrm{s}}}^{\mathrm{R}} \exp \left(\mathrm{j} n_{\mathrm{s}} \theta\right),
$$

where $n_{\min }$ and $n_{\max }$ are the minimum and maximum circumferential wave numbers, respectively, employed in the modal expansion of the reflected field. The second difference is that, unlike before, the number of constraint equations resulting from applying the continuity conditions on boundary $B^{+}$ does not generally equal the number of modes in the modal expansion so the system is not immediately invertible. Application of the principle of virtual work is applied to develop a system of equations from which the scattered waves' amplitudes are recovered straightforwardly. Then no further modifications are necessary. Further details are available in [1].

\section{ILLUSTRATIVE EXAMPLES}

\subsection{Overview}

Having provided an overview of the hybrid-SAFE technique, it is applied in this section to wave scattering from two illustrative rectangular notches in an otherwise blemish free pipe. The first notch is axisymmetric, while the second is nonaxisymmetric. Both notches are outer surface breaking and they have finite radial depths and axial extents. (Note that the accuracy of the software has been checked by applying transparency and energy balance considerations [1].) The properties assumed for the unblemished pipe and excitation pulse are described first. Then an overview is given of the SAFE analysis to recover approximate wave functions for the pipe. Finally results are given for the wave scattering from the two illustrative notches.

\subsection{Unblemished Pipe's Description}

The unblemished pipe, whose properties are summarized in Table 1, is assumed to be uniform, hollow, right circular, homogeneous, and isotropic. These properties are representative of an unblemished, seamless, Schedule 40, $80 \mathrm{~mm}$ diamètre nominal (DN), steel pipe. Moreover, the properties are essentially identical to the pipe examined experimentally in [5]. Where possible, (selected) direct comparisons between the experimental data given in [5] and the simulations presented here are also given. 


\subsection{Excitation's Form}

The function, $p(t)$, which describes the temporal variation of the applied force is idealized as the commonly used Gaussian modulated sine wave that has the form:

$$
p(t)= \begin{cases}0, & t<0 \\ A \exp \left[-a(s t-\tau)^{2}\right] \sin \left(s \omega_{0} t\right), & t \geq 0,\end{cases}
$$

where $A$ is an amplitude, $a$ determines the rate of decay of the pulse, $s$ serves to "scale" time, $\tau$ centres the pulse in time, $t$, and $\omega_{0}$ sets the centre frequency of the sine wave. The constant $a, s, \tau$, and $\omega_{0}$ are taken invariably to be:

$$
\begin{aligned}
& a=2.29595 \times 10^{10} \mathrm{~s}^{-2} ; s=0.28 ; \\
& \tau=1.4 \times 10^{-5} \mathrm{~s} ; \omega_{0}=\left(5 \times 10^{5}\right) \pi \mathrm{rad} / \mathrm{s} .
\end{aligned}
$$

Moreover, the (body force) amplitude is always $A=(\mu / H)$ and all nondimensionalized (body) forces are given with respect to $(\mu / H)$ ). (In the remainder of the text, a quantity embellished with a superscript asterisk indicates that it has been nondimensionalized.) The pulse is smooth (i.e., differentiable) in both time and frequency and, with the chosen constants, has a $70 \mathrm{kHz}$ centre frequency and over $99 \%$ of its energy is contained within the 35 to $107 \mathrm{kHz}$ bandwidth. Therefore the Fourier integral transform of $p(t)$, $\bar{p}(\omega)$, may be assumed reasonably to be contained within this finite bandwidth. The resulting forms of $p(t)$ and $|\bar{p}(\omega)|$, are illustrated in Fig. 2. Note that this excitation has been successfully employed experimentally, as in, for example, [1], [5], and [8].

Table 1. Properties assigned to the unblemished pipe

\begin{tabular}{lc}
\hline Property & Assigned value \\
\hline Density, $\rho,[\mathrm{kg} \mathrm{m}-3]$ & 7932 \\
\hline Outer diameter, $D_{\mathrm{o}},[\mathrm{mm}]$ & 88.8 \\
\hline Wall thickness, $H,[\mathrm{~mm}]$ & 5.59 \\
\hline Mean radius, $R,[\mathrm{~mm}]$ & 41.60 \\
\hline Thickness to mean radius ratio, $(H / R)$ & 0.134 \\
\hline Young's modulus, $E,[\mathrm{GPa}]$ & 216.9 \\
\hline Lamé constant $($ Shear modulus), $\mu(G),[\mathrm{GPa}]$ & 84.3 \\
\hline Lamé constant, $\lambda,[\mathrm{GPa}]$ & 113.2 \\
\hline Ratio of Lamé constants, $(\lambda / \mu)$ & 1.34 \\
\hline Poisson's ratio, $v$ & 0.286 \\
\hline
\end{tabular}

\subsection{Approximate Wave Functions from SAFE}

In determining approximate wave functions by using SAFE, ten identically thick finite elements are used to uniformly discretize the pipe's wall thickness, $H$. The circumferential angle, $2 \theta_{0}$, over which the spatial pulse approximates the Dirac delta function, is taken to be 0.002 radians $\left(0.1^{\circ}\right)$. Circumferential wavenumbers $n$, from 0 to \pm 16 , and all the $6 N+3$ a)

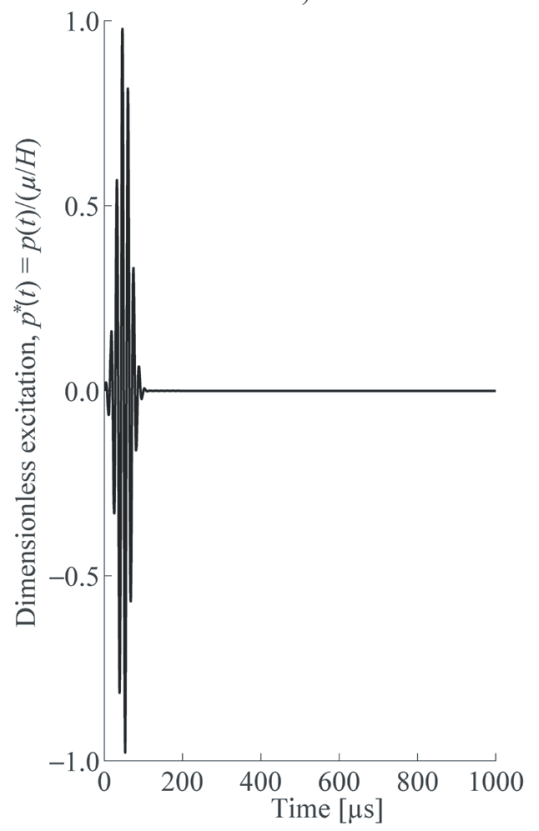

b)

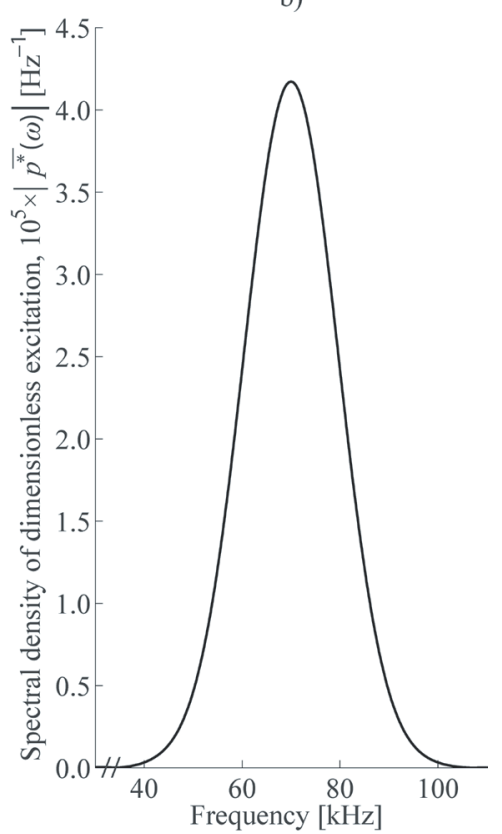

Fig. 2. Applied excitation in a) time and b) frequency 
corresponding axial modes are incorporated into the wave scattering computations. Numerical and experimental investigations of the unblemished pipe's displacement response may be found in [1] and [8].

\subsection{Axisymmetric Notch}

The axisymmetric notch has the dimensional properties summarized in Table 2. Eight node, quadratic axisymmetric finite elements [7] are utilized for the finite element region around the notch. A uniform idealization is selected after successfully checking the convergence of representative reflection coefficients [1]. Ultimately, ten (five) finite elements describe the behaviour over the wall thickness in the wave function (finite element) region. Furthermore four finite elements, which together correspond to half the notch's axial extent, are utilized axially. This selection allows longer axial notches to be represented without the need for additional axial finite elements. Note that the smallest propagating wavelength over the excitation's bandwidth is about $3.4 \mathrm{H}$, and belongs to the $\mathrm{L}(0,1)$ mode. Consequently, the ratio of the smallest (axial) wavelength excited to a finite element's axial length is approximately 48, almost five times larger than the (minimum) recommended guideline given by [5] of ten elements per shortest wavelength.

Table 2. Dimensions of the outer surface breaking, axisymmetric notch

\begin{tabular}{lc}
\hline Property & Assigned value \\
\hline Depth of notch, $d,[\mathrm{~mm}]$ & 2.79 \\
\hline Axial length of notch, $l,[\mathrm{~mm}]$ & 3.17 \\
\hline Depth to wall thickness ratio, $(d / H)$ & 0.500 \\
\hline Axial length to wall thickness ratio, $(l / H)$ & 0.568 \\
\hline
\end{tabular}

The transient point-like force is applied radially to the simulated pipe's outer surface at $z_{\mathrm{L}}{ }^{*}=\left(z_{\mathrm{L}} / H\right)=$ 5.1, where $z_{\mathrm{L}}$ is the transmitting transducer's axial coordinate. (Note that angles are measured relative to the idealized force's central point of application.) The resulting radial displacement is computed on the pipe's outer surface at $\theta_{\mathrm{R}}=0$, i.e., a pure axial offset from the point load, and $z_{\mathrm{R}}{ }^{*}=\left(z_{\mathrm{R}} / H\right)=10.2$, where $z_{\mathrm{R}}$ is the axial coordinate of the receiving transducer located in the reflected field. (The transmitted and reflected fields give similar information so the former is omitted.) All pertinent positions are shown in Fig. 1.

The (approximate) wave functions for the unblemished pipe were determined by using SAFE. Then the hybrid-SAFE technique was applied on a mode by mode and frequency by frequency basis. A modal superposition was applied at each frequency and the inverse Fourier transform was approximated using a numerical integration scheme to recover time histories from the approximate spectral densities. Fig. 3 shows typical displacement responses predicted in time and frequency for a pipe having no notch and a pipe having the outer surface breaking, axisymmetric notch. The displacement responses are evaluated on the pipe's outer surface where $\theta_{\mathrm{R}}=0$ and $z_{\mathrm{R}}{ }^{*}=\left(z_{\mathrm{R}} / H\right)=10.2$.

A cursory examination of Fig. 3 shows that the incident and reflected waves interact and cannot be separated in time. However, the notch's presence is discerned easily from a comparison of the corresponding spectral densities. This is because each predominant peak in the spectral density of Fig. 3b "splits" into two local maxima in Fig. 3d, one on either side of the original peak. The sharper maximum at the lower frequency also has a much larger amplitude so, for convenience, it is termed a "singularity." Differences between the frequencies of such singularities and the nearest cutoff behaviour of the unblemished pipe, having the frequencies shown in Fig. 3 b are presented in Table 3.

Table 3. Frequencies that correspond to the readily identified singularities appearing in Fig. $3 d$; they are distinct from the unblemished pipe's cutoff frequencies

\begin{tabular}{crcc}
\hline $\begin{array}{c}\text { Circumferential } \\
\text { wavenumber }\end{array}$ & $\begin{array}{c}\text { Axial } \\
\text { order }\end{array}$ & $\begin{array}{c}\text { Frequency of } \\
\text { singularity [kHz] }\end{array}$ & $\begin{array}{c}\text { Difference between } \\
\text { cutoff and singularity } \\
\text { frequency [kHz] }\end{array}$ \\
\hline$n \pm 8$ & $m=1$ & 43.112 & 0.085 \\
\hline \pm 2 & 3 & 46.462 & 0.001 \\
\hline \pm 4 & 2 & 49.623 & 0.294 \\
\hline \pm 9 & 1 & 52.983 & 0.129 \\
\hline \pm 10 & 1 & 63.362 & 0.192 \\
\hline \pm 11 & 1 & 74.153 & 0.273 \\
\hline \pm 12 & 1 & 85.274 & 0.377 \\
\hline \pm 13 & 1 & 96.660 & 0.503 \\
\hline
\end{tabular}

To help explain the frequency dependent behaviour of the notched pipe in the neighbourhood of the unblemished pipe's cutoff frequencies, Fig. 4 shows other normalized reflection coefficients predicted for the flexural $\mathrm{F}(n, 1)$ modes, where $n$ equals 8 through 13 , for an axisymmetric notch having $(d / H)=0.5$ and $(l / H)=0.568$. Each curve represents a single $\mathrm{F}(n, 1)$ mode which is reflected into itself. (Modal conversions from $\mathrm{F}(n, 1)$ into $\mathrm{F}(n, m)$, $m \neq 1$, are not given for brevity. Such conversions are required to satisfy continuity and boundary conditions.) For easier comparisons, the frequency 
a)

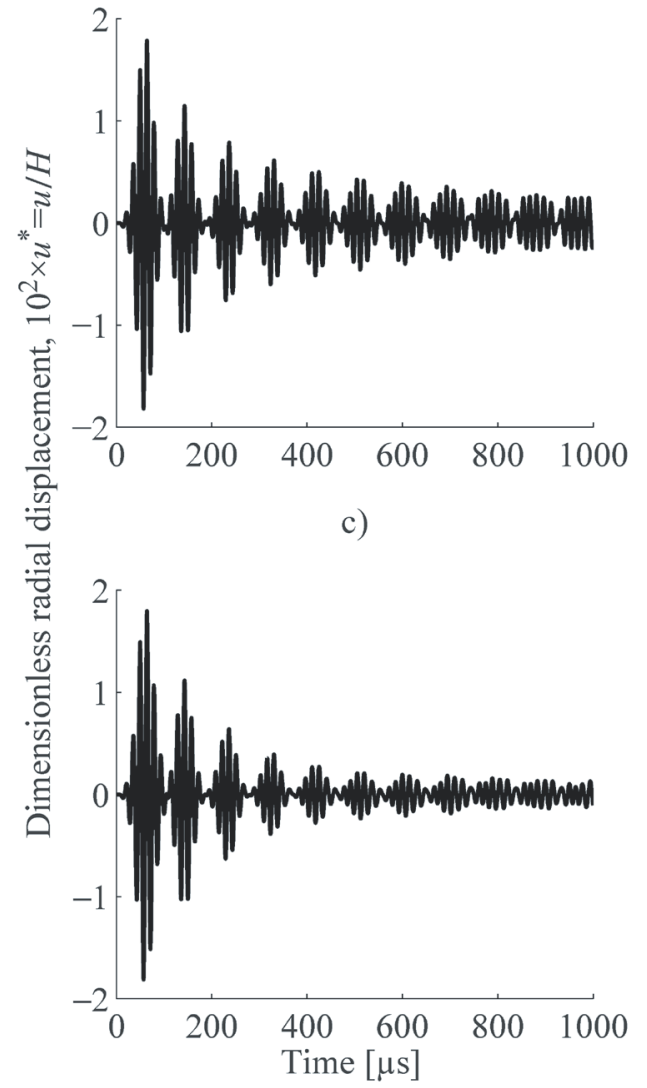

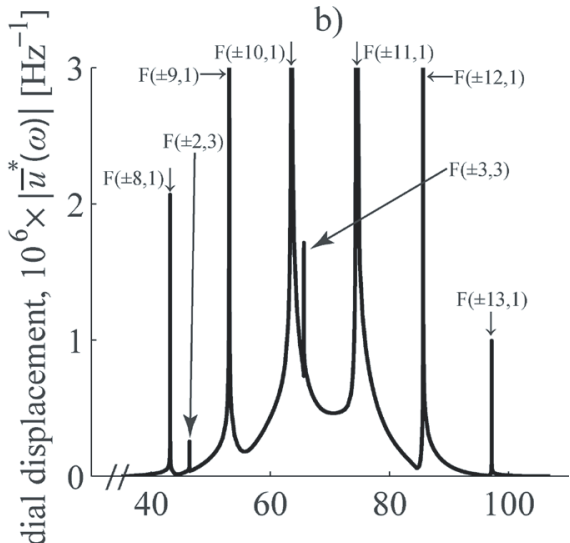

d)

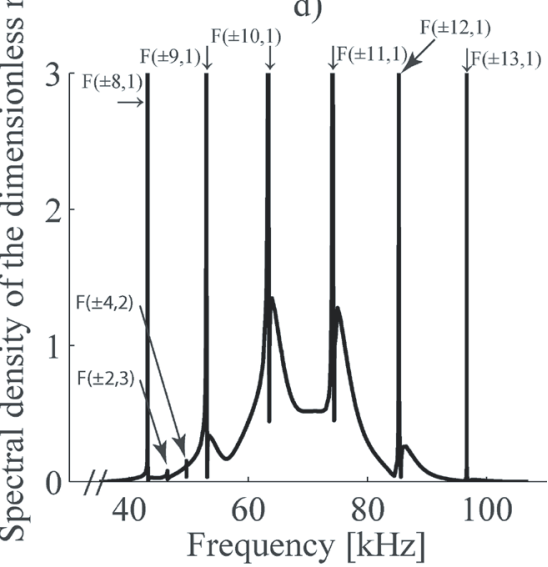

Fig. 3. Radial displacement predicted on the pipe's outer surface within the reflected field where $\theta_{\mathrm{R}}=0$ and $z_{\mathrm{R}}{ }^{*}=\left(z_{\mathrm{R}} / H\right)=10.2$ for an axisymmetric notch having $(d / H)=0.5$ and $(l / H)=0.568$; a) and b) direct waves produced by a radial point force; c) and d) superposition of direct and reflected waves produced by a radial point force

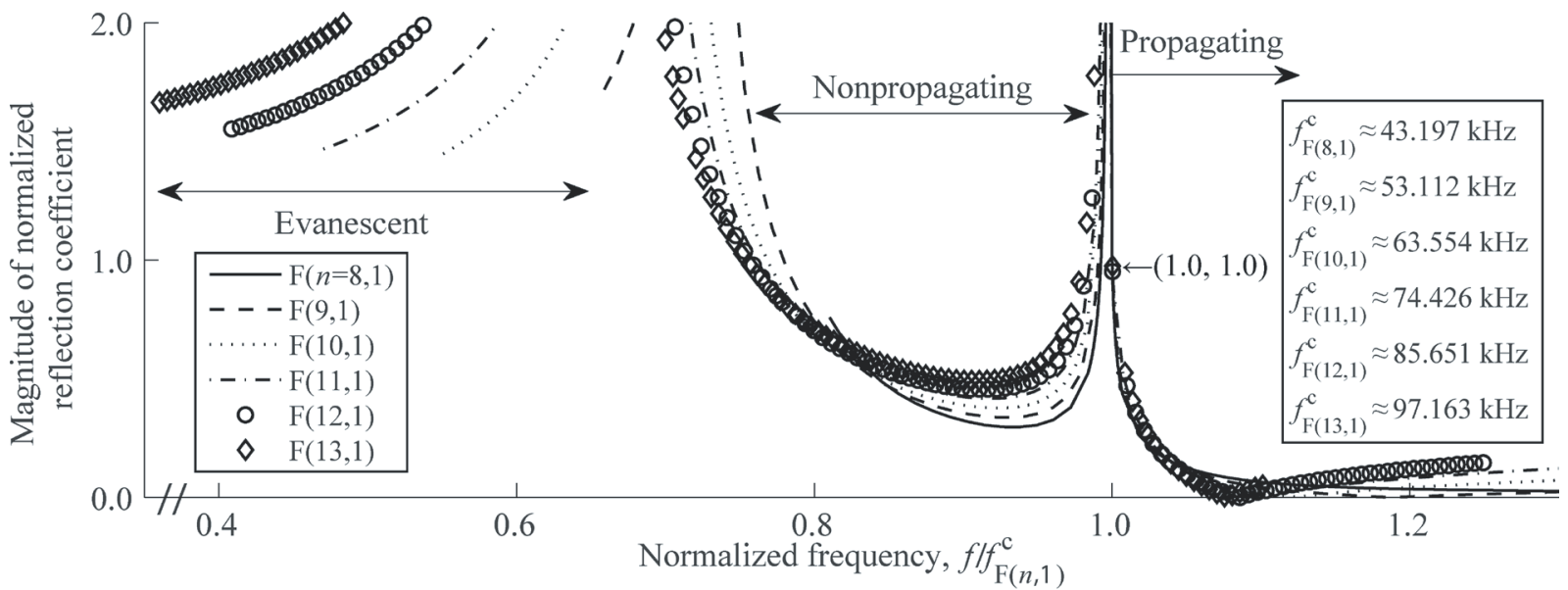

Fig. 4. Normalized reflection coefficient caused by flexural $\mathrm{F}(n, 1)$ modes, where $n$ is 8 through 13 inclusive, and an axisymmetric notch having $(d / H)=0.5$ and $(l / H)=0.568$; the $f_{\mathrm{F}(n, 1)}^{\mathrm{c}}$ is the cutoff frequency of the unblemished pipe's $\mathrm{F}(n, 1)$ mode

axis in Fig. 4 has been normalized by the cutoff frequency of the mode in question and expanded to lower frequency ratios. It is noteworthy now that each curve can be seen to possess two singularities. The singularity near a normalized frequency of 1.0 occurs, as before, at a frequency just below the 
unblemished pipe's relevant cutoff frequency. This observation can be corroborated by noting that the normalized reflection coefficients always pass through the point $(1.0,1.0)$ for these modes. On the other hand, the up-rise around the lower normalized frequency of 0.7 always corresponds to a mode transitioning from evanescent to non-propagating. The practical usefulness of this up-rise, however, may be limited. Waves scattered from the axisymmetric notch at frequencies near this up-rise decay exponentially from the notch's vertical boundaries at a rate of about $\exp \left(-z_{\mathrm{d}}{ }^{*}\right)$. The $z_{\mathrm{d}}{ }^{*}$ is the distance from the notch's boundary, nondimensionalized by the unblemished pipe's thickness, $H$. The decay rate in the axial direction is determined approximately based on the behaviour of the representative $F(10,1)$ mode's axial wavenumber found from Fig. 5. The latter figure shows that the imaginary part of the nondimensional axial wavenumber of this mode is almost one when the mode transitions from evanescent to nonpropagating. The quite large exponent suggests that the effect is very localized and likely to be masked by the propagating modes. The singularity just below the cutoff frequency of $63.553 \mathrm{kHz}$, on the other hand, is more interesting. Its effect is not so localized because the magnitude of the imaginary part of its wavenumber is much closer to zero. Indeed, Fig. 5 suggests that the axial decay rate is about $\exp \left(-0.15 z_{\mathrm{d}}{ }^{*}\right)$ at the notchinduced singularity. As a consequence of the smaller exponent, this last singularity is detectable further from the notch's vertical boundaries.

An analysis of the eigenvalues (resonant frequencies) of solely the finite element region (which can be found in [1]) indicates that the frequency of the possibly more important singularity does not correspond to a resonant frequency of the finite element region alone. It depends presumably upon the properties of both the finite element region and the parent waveguide. Moreover, the last column of Table 3 shows that the difference between the frequency of this singularity and the corresponding unblemished pipe's cutoff frequency grows continuously as the circumferential wavenumber increases. Advantage might be taken of this trend by increasing the centre frequency of the point force to excite modes having larger circumferential wavenumbers in order to make the frequency differences easier to measure.

Having examined the wave scattering from one particular notch, wave scattering by axisymmetric notches having various dimensions is considered. Fig. 6 presents the frequency difference (reduction), $\Delta f$, from a nearby cutoff frequency of the unblemished pipe caused by each notch. Results are shown for the $\mathrm{F}(10,1)$ mode but data for the $\mathrm{F}(11,1)$ and $\mathrm{F}(12,1)$

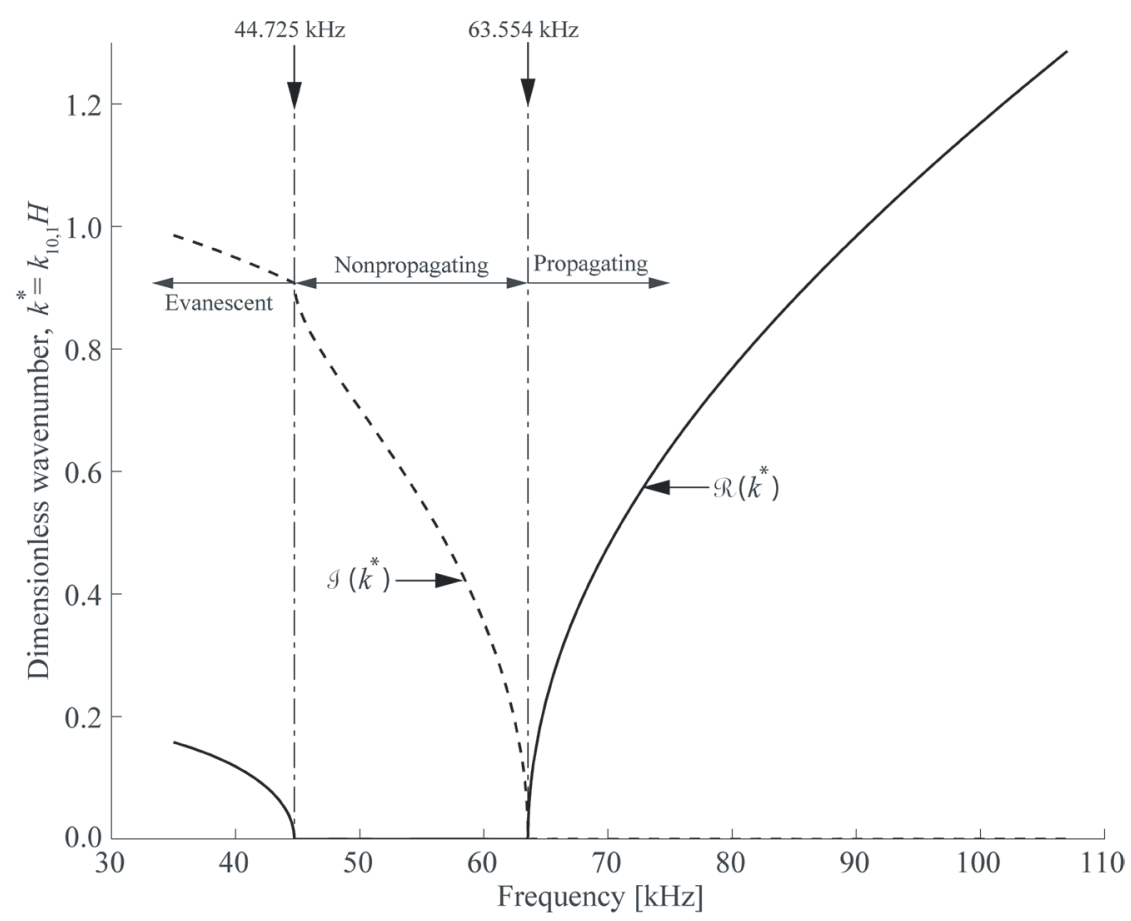

Fig. 5. Real and imaginary parts of the unblemished pipe's $F(10,1)$ nondimensional axial wavenumber, $k^{*}=k_{10,1} H$, as a function of frequency 


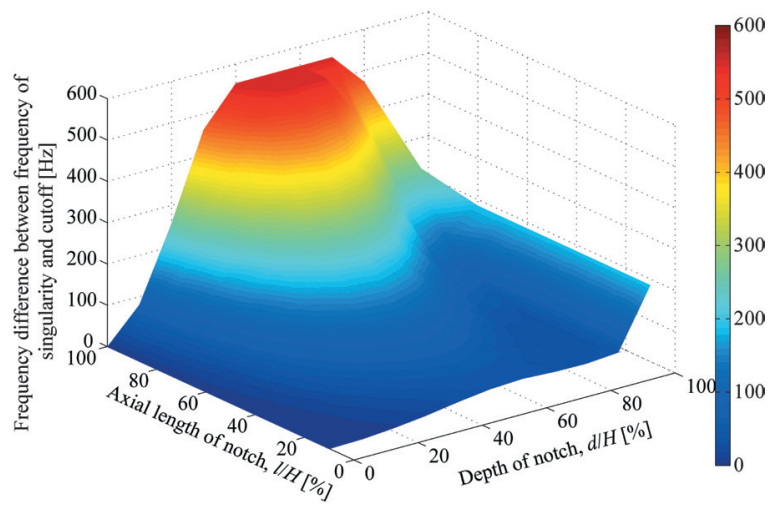

Fig. 6. Frequency differences, $\Delta f$ from the unblemished pipe's $F(10,1)$ cutoff frequency introduced by axisymmetric notches having various dimensions

modes are similar. Frequency reductions can be seen to depend upon an axisymmetric notch's depth and, to a less degree, its axial length. To determine these two dimensions, constant frequency differences are projected for each of the $\mathrm{F}(10,1), \mathrm{F}(11,1)$, and $\mathrm{F}(12,1)$ modes onto their common horizontal plane. These projections are superimposed in Fig. 7. Not surprisingly it can be seen that, due to the contours' "U-shapes," the depth ratio of a notch for a given axial length ratio, $(l / H)$, and constant frequency reduction, $\Delta f$, cannot be found absolutely from any single one of the three modes. Consequently more than one mode has to be employed - a situation which is common to a reflection based procedure [4].

The intersection of the contours of two different flexural modes is usually unique. See, for example, the $200 \mathrm{~Hz}$ and $300 \mathrm{~Hz}$ contours for the $\mathrm{F}(11,1)$ and $\mathrm{F}(12,1)$ modes, respectively. The single intersection of the contours provides two coordinates which uniquely define the two dimensions of an axisymmetric notch. Interpolations are obviously needed if a frequency difference does not lie precisely on a contour line.

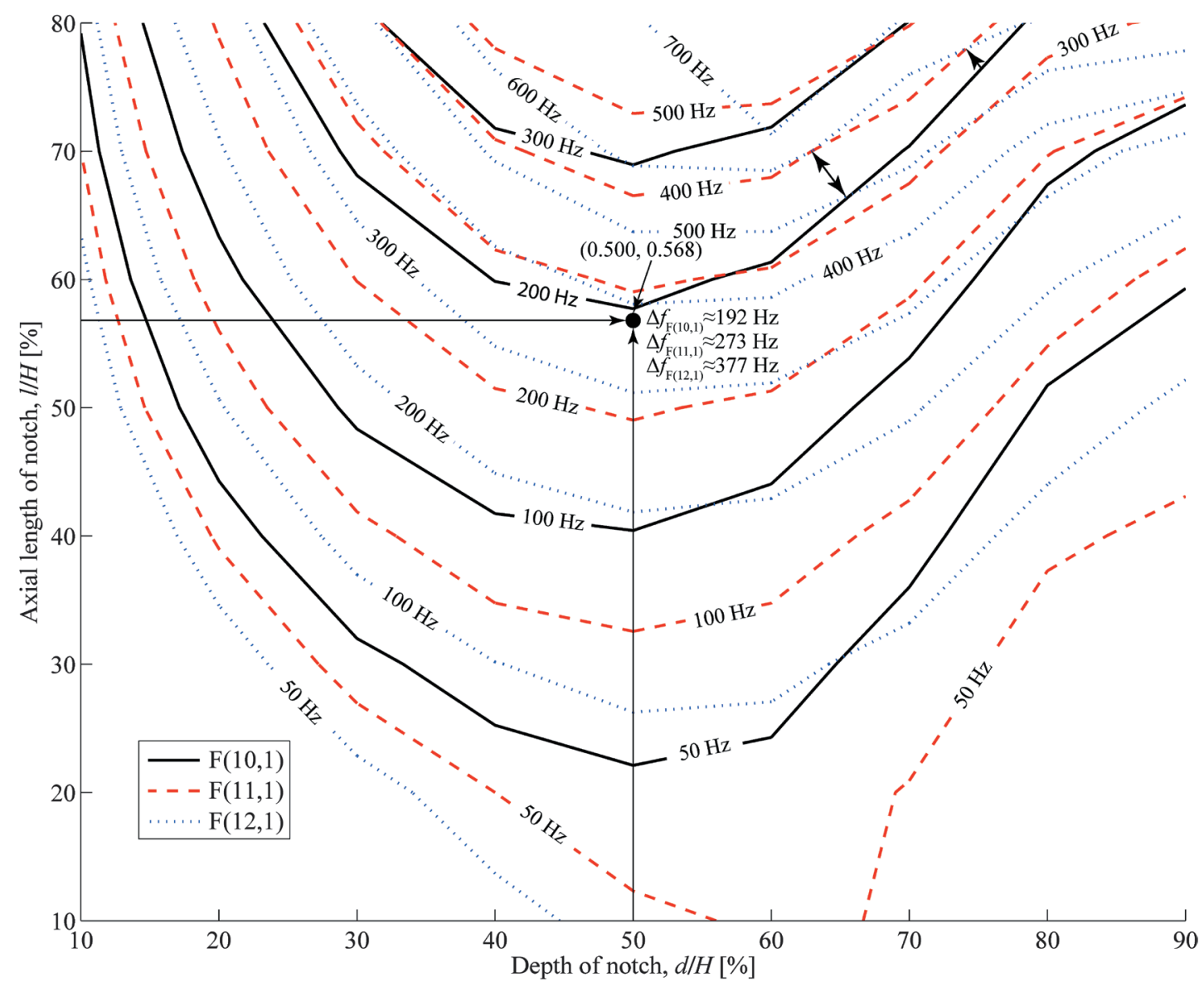

Fig. 7. Contour maps of constant frequency differences between the singularity produced by an axisymmetric notch and the unblemished pipe's cutoff frequencies for the $F(10,1), F(11,1)$, and $F(12,1)$ modes; The solid curves correspond to contours of the $F(10,1)$, dashed to $F(11,1)$, and dotted to $F(12,1)$ mode 
An example of this situation is illustrated in Fig. 7 where the frequency differences tabulated in Table 3 for the $F(10,1), F(11,1)$, and $F(12,1)$ modes are indicated. These differences can be used to uniquely characterise the notch's dimensions. Fig. 7 also suggests that, for a given frequency difference, a flexural mode with a higher circumferential wavenumber has a lower position. Consequently such modes are more sensitive to smaller notches. There are instances, however, when the curves for two different modes intersect more than once. One such example seen in Fig. 7 occurs for the $400 \mathrm{~Hz}$ and $600 \mathrm{~Hz}$ contours of the $\mathrm{F}(11,1)$ and $\mathrm{F}(12,1)$ modes, respectively. In this instance the two arrowed distances from the $\mathrm{F}(10,1)$ mode's $200 \mathrm{~Hz}$ reference contour may be used to distinguish the two intersections. Then, by interpolating linearly between the 200 and $300 \mathrm{~Hz}$ contours of the $\mathrm{F}(10,1)$ mode, a frequency difference in the $\mathrm{F}(10,1)$ mode of around $225 \mathrm{~Hz}$ would suggest a notch having $(d / H) \approx$ 0.73 and $(l / H) \approx 0.78$. On the other hand, a frequency difference of about $260 \mathrm{~Hz}$ in the $\mathrm{F}(10,1)$ mode would imply a notch with $(d / H) \approx 0.62$ and $(l / H) \approx 0.70$. Clearly, however, each additional intersection requires knowledge of another mode's frequency difference to uniquely determine a notch's dimensions. Furthermore, an excitation such as a point force which simultaneously excites several modes becomes more advantageous as the number of required modes increases.

\subsection{Nonaxisymmetric Notch}

The extension to nonaxisymmetric notches is considered now and the procedure for the axisymmetric notch is essentially followed. The reference nonaxisymmetric notch has dimensions identical to the axisymmetric notch considered earlier (see Table 2) with the exception that its circumferential extent is reduced to one-half of the unblemished pipe's circumference. Twenty-seven node, brick finite elements using quadratic Lagrange interpolation polynomials in each coordinate direction [7] were employed for the finite element region around the notch. The notch was modelled again by simply removing appropriate finite elements. Consequently the far field behaviour is meaningful. Ultimately, ten identically thick finite elements described the behaviour over a full wall thickness. To reduce computer waiting time, the minimally acceptable two finite elements always represented a notch's axial extent. However, 126 elements were deployed invariably around the pipe's unadulterated circumference. The radial discretization of ten elements through the unblemished pipe's wall was selected so that the wavefunctions from the previous axisymmetric analysis could be employed.

The appropriateness of the minimal axial discretization was checked by simulating the previous axisymmetric notch with the three-dimensional software. The two sets of reflection and transmission coefficients were each essentially indistinguishable. The circumferential discretization was determined, after selecting the radial and axial discretizations, by considering the results from transparency tests. The number of finite elements around the unblemished pipe's circumference was increased gradually until the reflection coefficient was less than 0.01 for all the modes propagating over some part of the excitation's bandwidth, 35 to $107 \mathrm{kHz}$ [1]. Therefore any reflection coefficient which has a magnitude greater than 0.01 for a propagating mode has an inconsequential error from this modelling component. Not surprisingly, the $F(13,1)$ mode dictated the circumferential discretization as it has the smallest (3.6H) circumferential wavelength of the propagating modes. On the other hand, the propagating $\mathrm{L}(0,1)$ mode has a somewhat smaller axial wavelength of around $3.4 \mathrm{H}$. Consequently, the ratio of the smallest axial wavelength of all the propagating modes to a finite element's axial length was approximately $12-\mathrm{a}$ value which is above the recommended lower bound of ten elements per shortest wavelength [5]. Similarly, the ratio of the $F(13,1)$ mode's (circumferential) wavelength to a finite element's circumferential length was virtually 10 .

Present and previous published [5] reflection coefficients, $\left|R_{\mathrm{L}(0,2), \mathrm{L}(0,2)}\right|$, are compared in Fig. 8 for different nonaxisymmetric notches. (Note that the finite element results in [5] are extrapolated by multiplying each result from a corresponding axisymmetric notch by the percentage ratio of the part circumferential notch length to the pipe's total circumference.) Solely the reflected $\mathrm{L}(0,2)$ component of the incident $\mathrm{L}(0,2)$ mode is considered. Note that a $50 \%$, circumferential notch is examined in Fig. 8a, while an 11\% circumferential notch is used in in Figs. $8 \mathrm{~b}$ and $\mathrm{d}$ so that direct comparisons can be made to the data given in [5]. Axial extents are varied more comprehensively, however, than before. Agreement is generally reasonable and $\left|R_{\mathrm{L}(0,2), \mathrm{L}(0,2)}\right|$ is seen in Fig. $8 \mathrm{~d}$ to grow not quite linearly with a notch's greater axial extent.

Fig. 9a is a comparable plot to Fig. 4 but with the $F(11,1)$ mode impinging solely on the nonaxisymmetric rather than the axisymmetric 
notch. The curves labelled $\mathrm{F}(11,1)$ in these figure have a similar overall character. Furthermore the frequency resolution in Fig. 9b, which lies an order of magnitude finer than the commonly employed 500 $\mathrm{Hz}$ here, appear to produce an additional singularitylike feature. It occurs between 74.30 and 74.35 $\mathrm{kHz}$; values which are again immediately below the $74.426 \mathrm{kHz}$ cutoff frequency of the $\mathrm{F}(11,1)$ mode for the unblemished pipe. Consequently the more detailed nature of the reflections from the two notches seems little different when the $\mathrm{F}(11,1)$ mode is considered alone. Conversely, Fig. 9a indicates that the nonaxisymmetric, unlike the axisymmetric, notch also converts the lone incident $\mathrm{F}(11,1)$ mode into superimposed reflections of principally the $\mathrm{F}(n, 1)$ modes having values of $n$ just below eleven. On the other hand, a comparison of Figs. 4 and $9 \mathrm{a}$ shows that each of the individual modal contributions retains almost all the prominent features observed for the axisymmetric notch. A computationally intensive frequency resolution, comparable to that used for $\mathrm{F}(11,1)$ in Fig. 9b, is still required however around singularities. Then the extension of the local procedure to dimensionalize an axisymmetric notch by using fairly small frequency differences can be explored. Furthermore, as propagating modes are also generated by nonaxisymmetric notches (through cross modal couplings), the possibility of remoter assessments could be also investigated.

\section{CONCLUSIONS AND CLOSING REMARKS}

A hybrid SAFE and standard finite element procedure was applied to detect and characterise an open notch in an infinitely long steel pipe. Axisymmetric notches were considered first. Interactions between incident and scattered guided waves and the axisymmetric notch were shown numerically to change a radial displacement's temporal history and introduce additional, singularity-like information in the
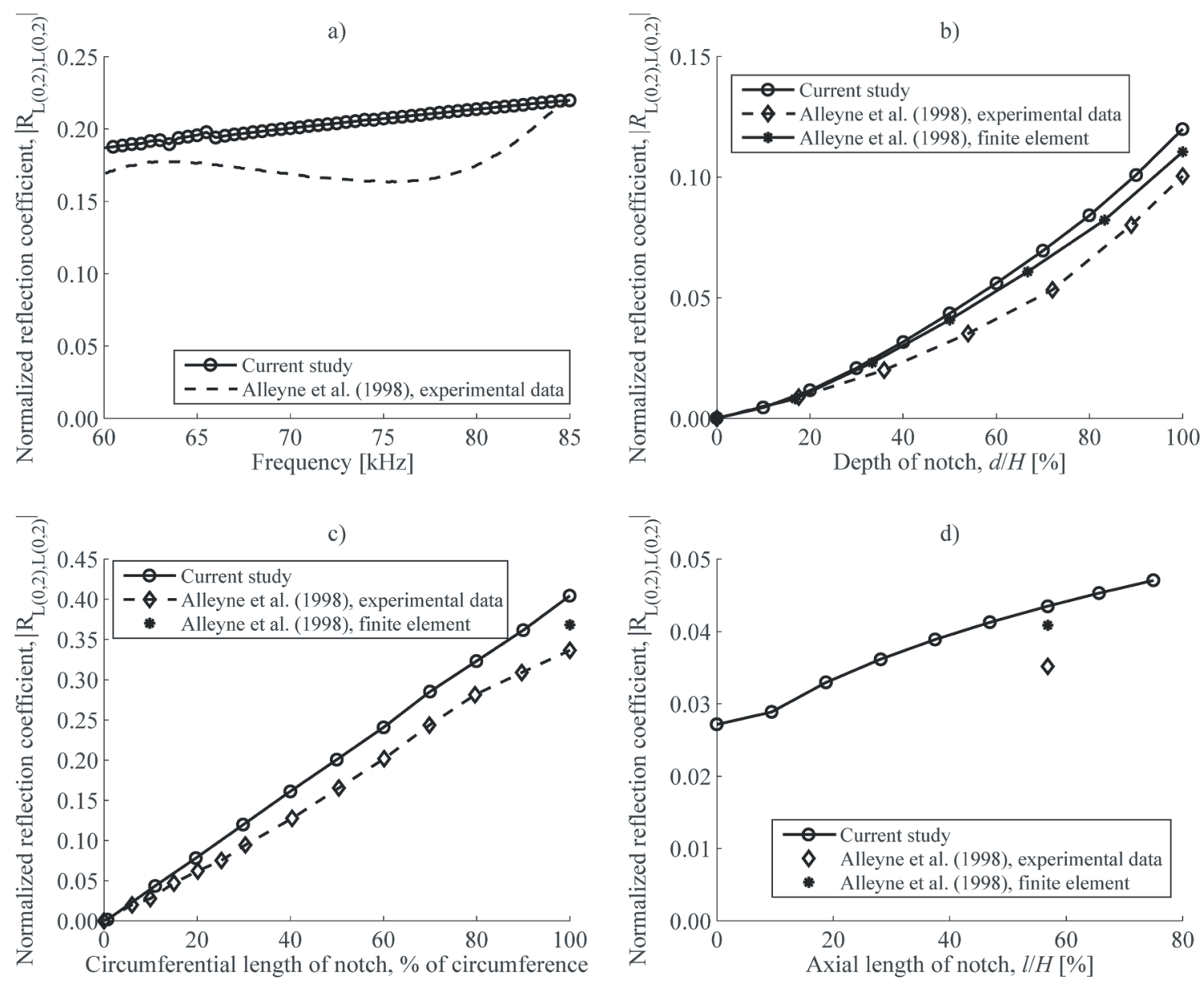

Fig. 8. Magnitude of the normalized reflection coefficient, $\left|R_{\mathrm{L}(0,2), \mathrm{L}(0,2)}\right|$, for different a) excitation frequencies, b) depths, c) circumferential extents, and d) axial lengths of nonaxisymmetric notches 
a)

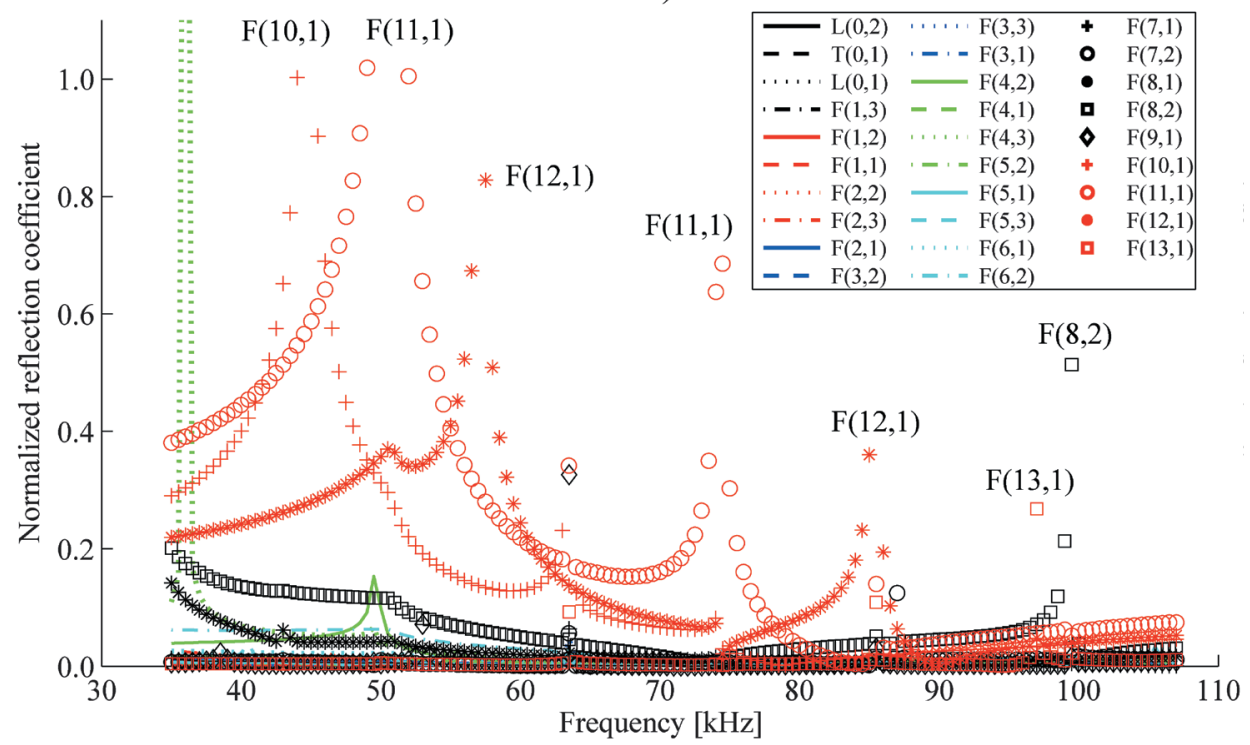

b)

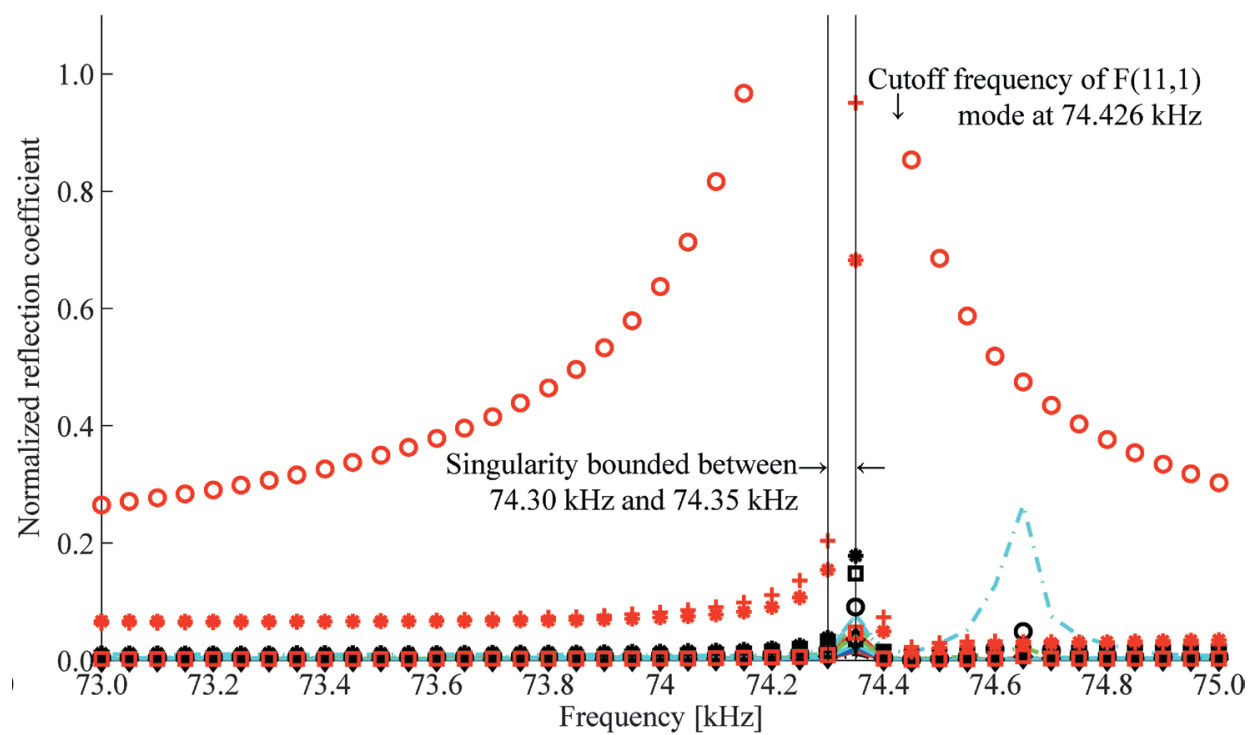

Fig. 9. Showing a) normalized reflection coefficients produced by the $F(11,1)$ mode incident on the reference nonaxisymmetric notch with $b)$ the frequency scale expanded about the $F(11,1)$ mode's cutoff frequency

previously unblemished pipe's frequency response function (FRF). This information indicated the presence of a nearby notch. Moreover, frequency differences between the "singularities" of the unblemished and blemished pipes were shown to reflect an axisymmetric notch's dimensions. A procedure by which an axisymmetric notch's dimensions could be estimated was demonstrated by considering the frequency differences for multiple modes. It is envisioned that, in practice, the experimental and signal processing techniques described in [8] could be utilized to simultaneously excite several modes and measure the resulting singularity frequencies. The extension to nonaxisymmetric notches was suggested.

The frequency differences are seen to grow with a larger circumferential wavenumber. Advantageous use might be made of this property by increasing the centre frequency of the excitation in order to excite modes with higher circumferential wavenumbers. However, effects are localized around the notch's axial boundaries because the frequencies of the singularities occur below nearby cut-off frequencies. This localization might be expected grow in size with a greater circumferential wavenumber because 
of the increasing frequency difference as a result of correspondingly increasing frequency differences. Because the technique applies only locally, it is unsuitable for rapidly screening large sections of pipe, unlike other complementary methods.

While no new experimental data is given, preliminary (and as of yet unpublished) experiments have shown that introducing a notch in a pipe generates singularities distinct from the pipe's cutoff frequencies. However, only "sharp," rectangular notches are considered. The extension to notches having different geometries still needs to be examined. Notwithstanding, the hybrid-SAFE approach can be applied straightforwardly to any arbitrary geometry providing that a finite element mesh suitably represents a notch's geometry.

The notches considered here are larger than those of practical interest, but serve to illustrate the proposed method. It is speculated, based on the limited data obtained to date, that singularities distinct from the unblemished pipe will be generated by notch-like defects of any dimensions. It is yet to be determined, however, if the frequency differences of small notches can be measured with sufficient precision to be useful.

\section{ACKNOWLEDGEMENTS}

All three authors acknowledge the financial support from the Natural Science and Engineering Research Council (NSERC) of Canada. The first author also wishes to acknowledge financial aid from the University of Manitoba Students' Union (UMSU), Society of Automotive Engineers (SAE) International, University of Manitoba, Province of Manitoba, and Ms. A. Toporeck and family. The Wawanesa Mutual Insurance Company is thanked for their generous donation of goods in kind. The authors wish also to thank Dr. Joseph L. Rose, Paul Morrow
Professor of Engineering Design and Manufacturing, of the Pennsylvania State University for the useful suggestions he made as the external examiner for the first author's doctoral dissertation. The experimental efforts of Mr. Kazeem Adeogun are recognized.

\section{REFERENCES}

[1] Stoyko, D. (2012). Using the Singularity Frequencies of Guided Waves to Obtain a Pipe's Properties and Detect and Size Notches. Ph.D. Thesis, University of Manitoba, Manitoba, from http://hdl.handle. net/1993/9818.

[2] Shin, H., Rose, J. (1998). Guided wave tuning principles for defect detection in tubing. Journal of Nondestructive Evaluation, vol. 17, no. 1, p. 27-36, DOI:10.1023/A:1022680429232.

[3] Oliver, J. (1957). Elastic wave dispersion in a cylindrical rod by a wide-band short-duration pulse technique. Journal of the Acoustical Society of America, vol. 29, no. 2, p. 189-195, DOI:10.1121/1.1908824.

[4] Demma, A. Cawley, P., Lowe, M., Roosenbrand, A.G., Pavlakovic, B. (2004). The reflection of guided waves from notches in pipes: A guide for interpreting corrosion measurements. NDT and E International, vol. 37, no. 3, p. 167-180, DOI:10.1016/j.ndteint.2003.09.004.

[5] Alleyne, D., Lowe, M., Cawley, P. (1998). Reflection of guided waves from circumferential notches in pipes. Journal of Applied Mechanics, vol. 65, no. 3, p. 635641, DOI:10.1115/1.2789105.

[6] Silk, M., Bainton, K. (1979). Propagation in metal tubing of ultrasonic wave modes equivalent to Lamb waves. Ultrasonics, vol. 17, no. 1, p. 11-19, DOI:10.1016/0041-624X(79)90006-4.

[7] R. Cook, (1981). Concepts and Applications of Finite Element Analysis. John Wiley and Sons, New York.

[8] Stoyko, D., Popplewell, N., Shah, A. (2010). Finding a pipe's elastic and dimensional properties using ultrasonic guided wave cut-off frequencies. NDT and $E$ International, vol. 43, no. 7, p. 568-578, DOI:10.1016/j. ndteint.2010.05.013. 